\title{
ASTEF: A simple tool for examining fixations
}

\author{
Marco Camilli, Roberto Nacchia, Michela Terenzi, and Francesco Di Nocera \\ University of Rome “La Sapienza,” Rome, Italy
}

\begin{abstract}
In human factors and ergonomics research, the analysis of eye movements has gained popularity as a method for obtaining information concerning the operator's cognitive strategies and for drawing inferences about the cognitive state of an individual. For example, recent studies have shown that the distribution of eye fixations is sensitive to variations in mental workload - dispersed when workload is high, and clustered when workload is low. Spatial statistics algorithms can be used to obtain information about the type of distribution and can be applied over fixations recorded during small epochs of time to assess online changes in the level of mental load experienced by the individuals. In order to ease the computation of the statistical index and to encourage research on the spatial properties of visual scanning, A Simple Tool for Examining Fixations has been developed. The software application implements functions for fixation visualization, management, and analysis, and includes a tool for fixation identification from raw gaze point data. Updated information can be obtained online at www .astef.info, where the installation package is freely downloadable.
\end{abstract}

This article describes the capabilities of $A$ Simple Tool for Examining Fixations (ASTEF), an offline data analysis application for processing eye-movement information. The tool was developed for specific needs in human factors and ergonomics (HF/E) research, because the analysis of individuals' visual behavior has become increasingly popular in this field.

The path drawn by the eyes during the exploration (or scanning) of a scene has been dubbed scanpath (Noton $\&$ Stark, 1971) - a sequence of successive fixations and saccades (between fixations). Seminal work (e.g., Ellis \& Smith, 1985; Ponsoda, Scott, \& Findlay, 1995; Stark \& Ellis, 1981) has been carried out for obtaining information about the visual search strategies that are possibly linked to variations in task demands. However, these studies have failed to show any computable effect of task complexity on visual scanning behavior. Nevertheless, the analysis of scanpath is still popular (e.g., Diez et al., 2001; Van Orden, Limbert, Makeig, \& Jung, 2001) and is considered to be a valuable method for obtaining information concerning the operator's strategies (e.g., about the areas of an interface that are inspected). Also, as recently stated by McCarley and Kramer (2007), oculomotor data can be used to draw inferences about the cognitive state or the level of mental workload of an individual.

Some studies have explored the ocular response in novice and expert participants involved in specific tasks. Particularly, shorter and more frequent fixations were recoded in expert operators (Bellenkes, Wickens, \& Kramer, 1997; Miller, 1973), whereas more frequent fixations, with less consecutive time spent on the same object (shorter dwell time), were found in expert pilots during a landing flight phase (Kasarskis, Stehwien, Hickox, Aretz, \& Wickens,
2001). These findings showed that visual scanning characteristics could be affected by expertise and suggested that the study of scanpath features could be useful for discriminating good from poor performance.

Other studies (Bunecke, 1987; Ephrath, Tole, Stephens, \& Young, 1980) have shown fixation duration to be affected by workload variations. Provisional ocular metrics of mental workload that are based on the analysis of scanpath were recommended over two decades ago (Ephrath et al., 1980; Harris, Glover, \& Spady, 1986; Tole, Stephens, Vivaudou, Ephrath, \& Young, 1983). Visual scanning randomness (or entropy) was found to be related to mental workload: High task load conditions would generate less randomness than would low task load conditions. In other words, transitions of fixations between different areas of interest (AOIs) were reduced when mental workload was high, indicating attentional narrowing.

For decades, this finding has been reported as a fact, despite there having been only a few articles (e.g., Hilburn, Jorna, Byrne, \& Parasuraman, 1997) reporting similar results. Moreover, there is also evidence of an opposite pattern, namely that higher entropy could be associated with higher mental workload as well (Kruizinga, Mulder, $\&$ de Waard, 2006).

In recent studies from our laboratory (Camilli, Terenzi, \& Di Nocera, 2007; Di Nocera, Camilli, \& Terenzi, 2007; Di Nocera, Terenzi, \& Camilli, 2006), we used a different perspective for investigating the relationship between mental workload and fixation distributions. The type of spatial distribution produced by the fixations' pattern was computed with a spatial statistics algorithm called the nearest neighbor index (NNI; Clark \& Evans, 1954). 
In the earliest of the studies above (Di Nocera et al., 2006), participants were engaged in two task conditions (easy and difficult) of the Asteroids arcade game. In this case, the nature of the tasks that were displayed was dynamic; that is, the visual components of the scene (the ship and the asteroids) constantly moved across the screen. An additional study (Di Nocera et al., 2007) involved 10 professional pilots who were requested to perform a simulated flight across five different phases (departure, climb, cruise, descent, landing). The visual scanning behavior was analyzed on a static-displayed scene (the cockpit instrument panel). In both studies, fixations appeared to be (near randomly) dispersed when workload was high, and grouped when workload was low. The validity of the NNI as a measure of mental workload was addressed in a subsequent methodological study (Camilli et al., 2007). In this case, participants were required to perform at different difficulty levels of the Tetris game. The NNI was contrasted with two concurrent measures-P300 and the NASA task load index (NASA-TLX) - frequently used as mental workload indicators. The results showed consistency between subjective (NASA-TLX scores), psychophysiological (P300 amplitude), and ocular (NNI) indexes.

Given its stability and validity, the spatial statistics account appears to serve as satisfactorily as does the entropy account. There are also reasons to actually prefer the former to the latter. Entropy needs a priori definition of AOIs, whereas spatial analysis eliminates this rather subjective element, and many studies have addressed the analysis of scanpath using a narrow field of view in which many features of the scene might be accessed peripherally. Although the term scanpath is often informally used to describe any eye-movement recording, Noton and Stark (1971) specifically state that a scanpath is obtained thusly: "If patterns are presented to a subject under conditions of poor visibility, so that he is forced to look directly (foveally) at each feature to which he wishes to attend, then the position of his eyes will reveal the features processed and his saccadic eye movements from feature to feature will reveal the order of processing" (p. 308). Obviously, conditions for studying the sequence of fixations do not hold in other cases. Moreover, in contrast to entropy calculation, spatial analysis takes into account all fixations (without loss of information), considering the fixation points' coordinates to be valuable sources for establishing how they are spatially distributed. Furthermore, the NNI can be computed over small epochs of 1 or 2 min, providing information about the temporal aspects of scans and allowing the monitoring of the functional state of an individual (see Camilli et al., 2007; Di Nocera et al., 2007).

Although more work has to be done in order to understand the functional significance of the relationship between fixation distribution and mental workload, it is plausible that it might reflect the use of different visual scanning strategies. Indeed, in complex and more demanding task situations, a wider fixation pattern (i.e., random or near random) might be used to optimize prompt attending to incoming information. This account makes sense, considering that, in the studies reported above, the prin- cipal variations in task demands were due to more stringent temporal constraints: either rapidly executing many checks or quickly performing many operations.

In agreement with that, Di Nocera and Bolia (2007) recently proposed that two processes might respectively contribute to dispersion and grouping in visual scanning behavior: temporal demand and visuospatial demand. Indeed, whereas studies that have reported grouping (not necessarily using entropy analysis; see Recarte \& Nunes, 2000) have challenged a visual primary task (e.g., driving or air traffic control) by using a secondary task that claimed resources from the very same pool (e.g., mental imagery, additional aircraft to control), studies reporting dispersion have devised task load conditions by manipulating the temporal demand (e.g., brisk stimulation).

Both accounts may then be valid. Nevertheless, a critical issue for comparing different study perspectives is the standardization of the computational practices for deriving ocular metrics of mental workload. With that in mind, we have developed ASTEF, which will eventually permit other scientists to fully test different hypotheses in order to investigate the relationship between visual scanning behavior and mental workload.

\section{INTRODUCING ASTEF}

Eyetracker manufacturers often provide software applications for playing back and analyzing the eye-movement data that are recorded by the system. Most of these applications offer several interesting features-sometimes many more than those required by the investigators using them. Also, the great number of functions makes these applications resource consuming and way too complicated for rapid and easy manipulation of coordinate data. In contrast, ASTEF development has been aimed at having a small application coded in C\#.NET and running on Microsoft Windows machines, allowing specific analyses of ocular data in a bidimensional space. The following sections will introduce three main capabilities of this simple tool: visualization, analysis of fixation data (AOI and fixation distribution), and fixation identification.

\section{Visualization}

Qualitative inspection of the sequence of fixations is one of the first tasks carried out by researchers working with ocular data. That might serve either as a checking procedure for assessing the quality of the recording or as a method for addressing the objective of the study (e.g., defining AOIs).

ASTEF permits visualization of the recorded scanpath in several different ways:

1. visualizing the succession of the fixations on the screen in pixel coordinates (using the customizable axes and grids or hiding them);

2. superimposing the scanpath on to a scanned scene (e.g., a snapshot of the visual task scenario);

3. replaying the scanpath concurrently with other recorded features (i.e., temporal interval between successive fixations and duration of each fixa- 


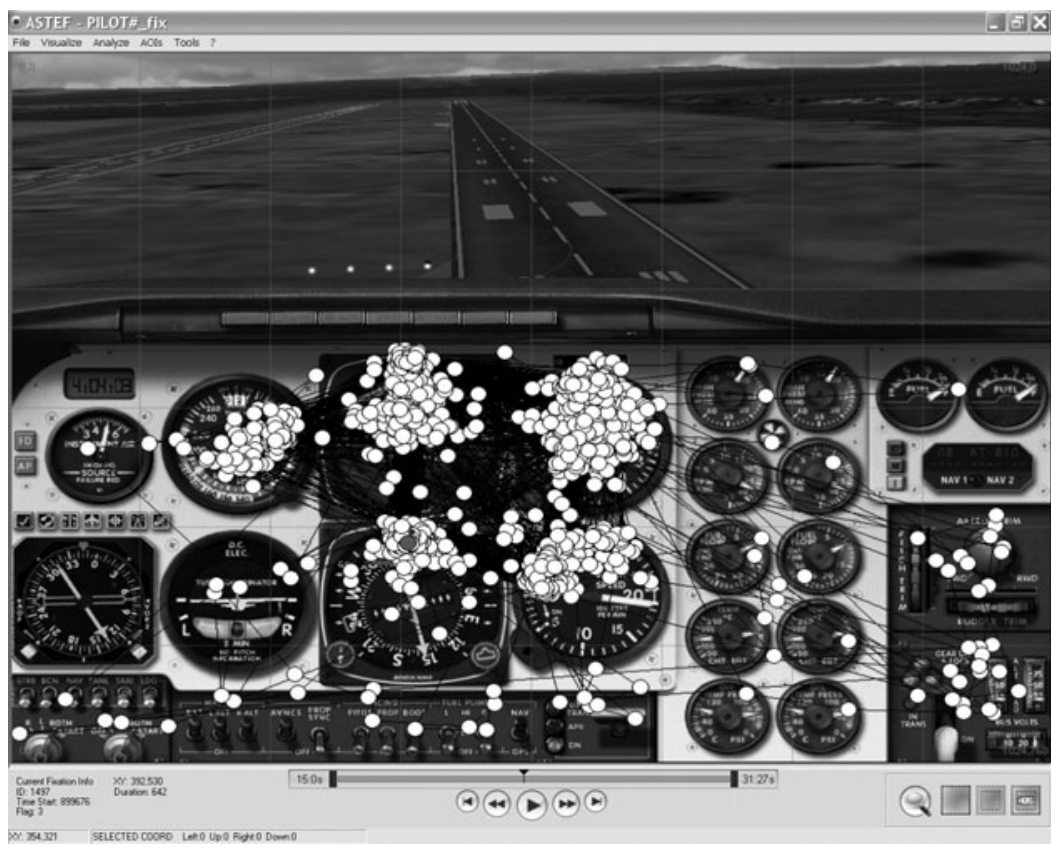

Figure 1. Example of scanpath visualization over a snapshot of a task scene.

tion) by clicking over the progress bar located at the bottom of the interface; or

4. scrolling the fixation sequence rapidly (forward or backward).

The default starting time of scanpath playback is the beginning of the recording session. However, a particular instance in time (or a given fixation number) can also be reached either by using the "Go To" function to click over the magnifier icon located at the bottom right-hand corner of the interface or by dragging the slider on the progress bar from side to side. Figure 1 shows a sample scanpath superimposed onto a snapshot of the task interface.

ASTEF groups most of the visualization functions in the "File" menu, which allows the loading, exporting, and saving of the scanpaths. By default, visualization includes grid, points, lines, and background images (if available). The "Visualize" menu allows rapid customization of the view. Also, by clicking over fixation points, saccades lines, and grids, it is possible to customize line thickness, circle radius, and colors.

In order to visualize the scanpath, a properly formatted input file must be opened. ASTEF input files must include the following information:

1. area size - the area in which the scanpath evolves (e.g., 1,024 × 768 pixels);

2. fixation recording start time - timing information that might be useful for data sync;

3. fixation duration-duration of the fixation, in milliseconds;

4. fixation coordinates - pixel coordinates $(x, y)$ defining the fixation position in the area (origin is located at the upper left-hand corner); and
5. flag-optional information that can be added by the experimenter for defining the experimental condition to which the fixation belongs.

Given that any eye-tracking system provides eye coordinates and timing, it is easy to edit any ASCII tab-separated data file in order to make it readable by ASTEF. Sample input files are available in the default folder (\astef 1.2 datalinput $\backslash$ samples).

Any graphic file format can be imported as background. This function is quite useful for playing the scanpath back over a meaningful picture in order to derive information about the participants' ocular behavior. For example, when a participant interacts with a software interface and the scanpath is subsequently played back over a screenshot of the very same interface, it is easy to examine the location of most of the fixations and the type of strategy used.

Scanpaths can be exported as ASCII data files (ASTEF formatted), static images, and video clips (Figure 2). The video exporting function uses Microsoft Windows application programming interfaces. In order to export animated scanpaths in the desired format, users can use their system's video codecs (e.g., Microsoft MPEG and Intel Indeo) or install those they prefer for customizing the speed and quality of video compression (e.g., DivX and Xvid).

AOI analysis. Analysis of temporal dynamics of the scanpath is one of the primary tasks accomplished by ASTEF. This is a common task for researchers who need to examine the progression of fixations in order to identify AOIs. In several studies (e.g., Diez et al., 2001; Hilburn et al., 1997), the definition of the AOIs was based on a priori criteria, taking into consideration what were assumed to be the most informative interface regions. These are considered by the researcher to be the necessary visual regions to be viewed by participants for performing a specific task. 


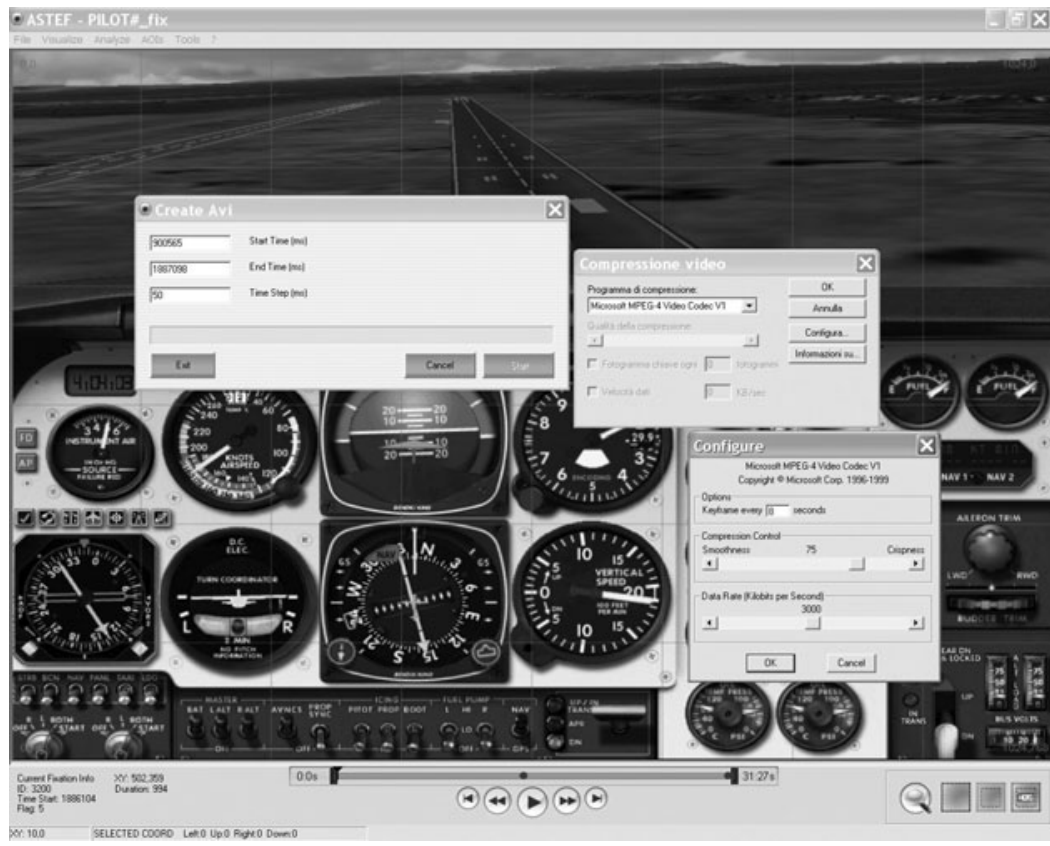

Figure 2. Example of scanpath clip exporting. Create AVI: Setup of start time and end time of recording. Compressione video: the choice of the codec for video compression. Configure: the configuration of the codec parameters.

In contrast, other researchers (e.g., Salvucci \& Goldberg, 2000) used a different approach for defining an AOI: the observation of fixations on a visual target and area. In other words, the recording of either frequent fixations or of their longer duration on the same visual area marks that area as being an AOI. In either case, the selection of an AOI is easily accomplished by using ASTEF, which implements area selection on visualized scanpath in three different ways:

1. dragging the diagonal of a rectangle (Figure $3 \mathrm{~A}$ );

2. moving the four sides of the rectangle separately, dragging the four corresponding cursors with the use of the mouse (Figure 3B); or

3. clicking on the "Input Area Selection Coord" icon (icon array at the bottom right-hand corner) and inserting the exact coordinates manually in the "Manual Selection Area" form (Figure 3C).

ASTEF also provides the capability to invert selections (Figure 3D). This is useful for operating on points outside an AOI (e.g., deleting all the points outside the AOI). During the selection procedures, the area size and mouse pointer coordinates are always visible in the status bar. All the selected AOIs can be named and saved for further use from the "AOIs" menu.

The "Analyze" menu has two functions- "fixation inspection" and "fixation duration"- that provide basic scanpath information including the following:

1. number of fixations;

2. earliest and latest fixation (ID and position in the timeline);

3. shorter and longer fixation (ID and duration); and
4. mean, standard deviation, and median of fixation duration.

This information is also accessible by right-clicking over either the whole scanpath or the single AOI.

\section{Fixation Distribution Analysis}

The present release of ASTEF (as of this article's publication) implements the NNI (or $R$, as it was originally introduced) as a measure of spatial dispersion (Figure 4). As reported by Clark and Evans (1954, p. 447), "distance from an individual to its nearest neighbor, irrespective of direction, provides the basis for this measure of spacing." This approach may be applied to any point pattern, and it was indeed applied to distributions of plants and animals. Given that fixations are point patterns as well, fixation distributions can also be analyzed using this method. In fact, if, in a set of $N$ fixations having a specified density $\rho$ (the number of fixations per unit of area), we measure the distance $r$ from each fixation to its nearest neighbor, the mean observed distance may be represented as

$$
\bar{r}_{A}=\frac{\sum r}{N} \text {. }
$$

The mean distance that would be expected if this set of fixations were distributed at random, $\bar{r}_{\bar{E}}$, can be shown to have a value equal to

$$
\frac{1}{2 \sqrt{\rho}}
$$

(demonstration is available in Clark \& Evans, 1954, pp. 451-452). The ratio 
A

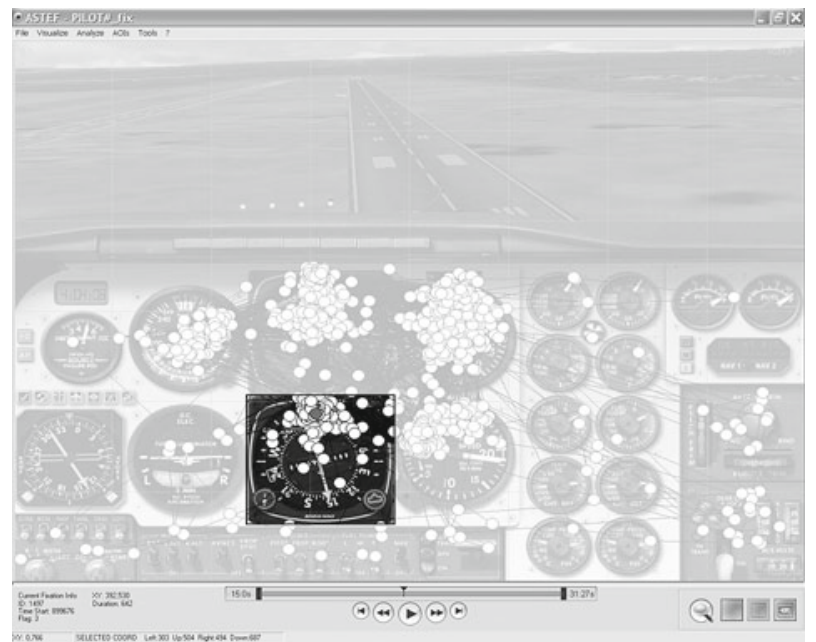

\section{c}

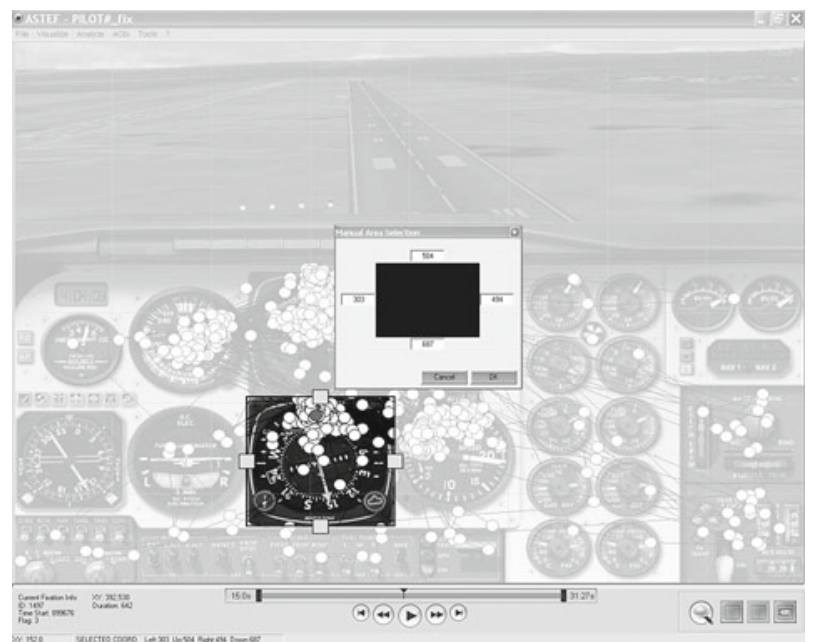

B

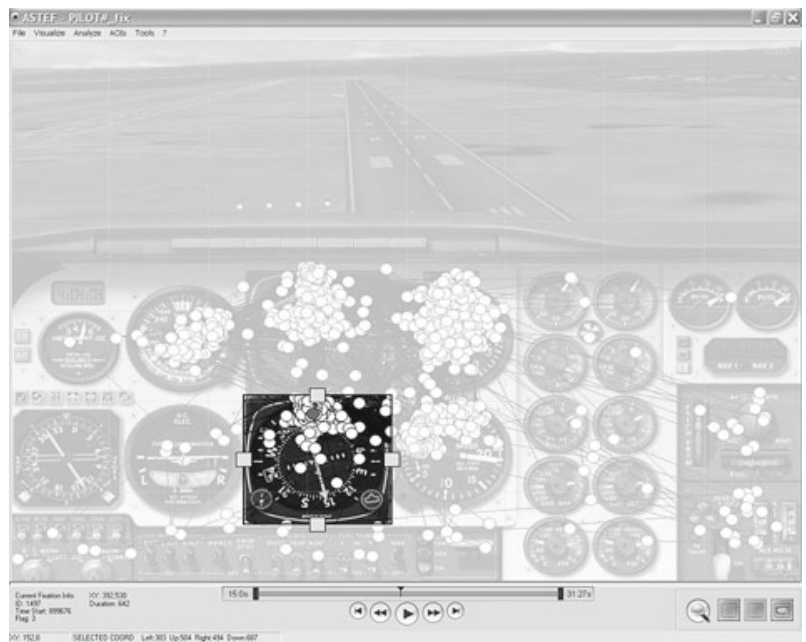

D

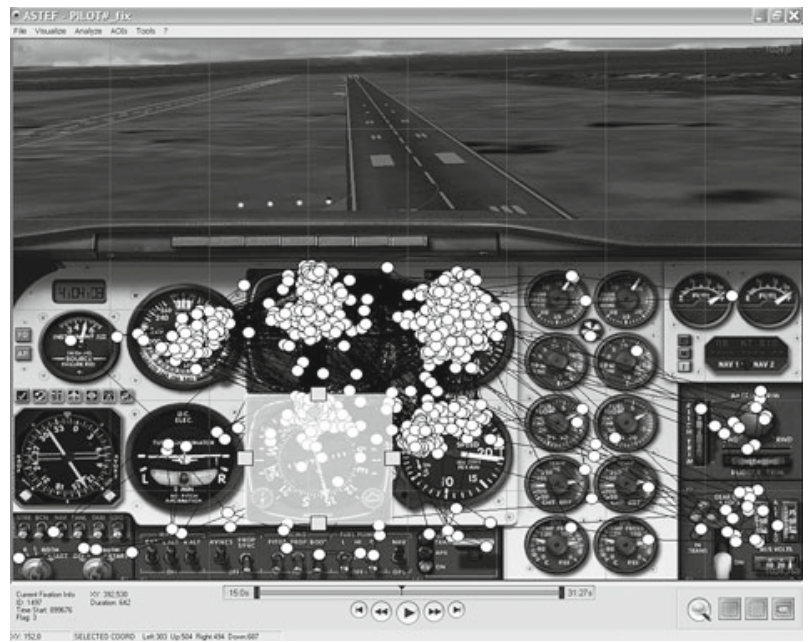

Figure 3. Different ways to select areas of interest in ASTEF. See text for explanation.

$$
R=\frac{\bar{r}_{\bar{A}}}{\bar{r}_{\bar{E}}}
$$

can then be used as a measure of the degree to which the observed distribution approaches or departs from random probability. Therefore, this procedure provides a single value that is indicative of the type of distribution on which it has been computed. As reported above, the mean distance between pairs of nearest neighbors is compared with that expected on the basis of chance. The actual mean distances can be smaller (points are aggregated; $R<1$ ), larger (points are regularly dispersed; $R>1$ ), or not different from the expected distances (points are randomly dispersed; $R=1$ ). The statistical significance of this index can also be derived, but, for the purposes of its application to the measure of mental workload, only the "raw" index has been used so far.

In ASTEF, it is possible to calculate $R$ either on the whole screen area or on a selected AOI (see Figure 4). The latter permits the consideration of collected fixations that occur on one region of the screen. After the determination of the screen analysis region, ASTEF permits the use of two different types of area (processed in the $R$ algorithm) for computing the NNI: convex hull $(\mathrm{CH})$ and smallest rectangle (SR). $\mathrm{CH}$ is derived by Delaunay's algorithm (Delaunay, 1934), which creates a temporary hull from the first three points, and then adds other triangles for each outer point (Figure 5A). SR is based on an algorithm that creates a bounding box for defining the rectangle that has the smallest area comprising all the examined points (Figure 5B).

For the CH, ASTEF also implements the Donnelly's edge effect adjustment method (Donnelly, 1978). Donnelly's correction is obtained by adding the following to $\bar{r}_{\bar{E}}$ :

$$
\left(0.0514+\frac{0.041}{\sqrt{N}}\right) \times \frac{B}{A},
$$




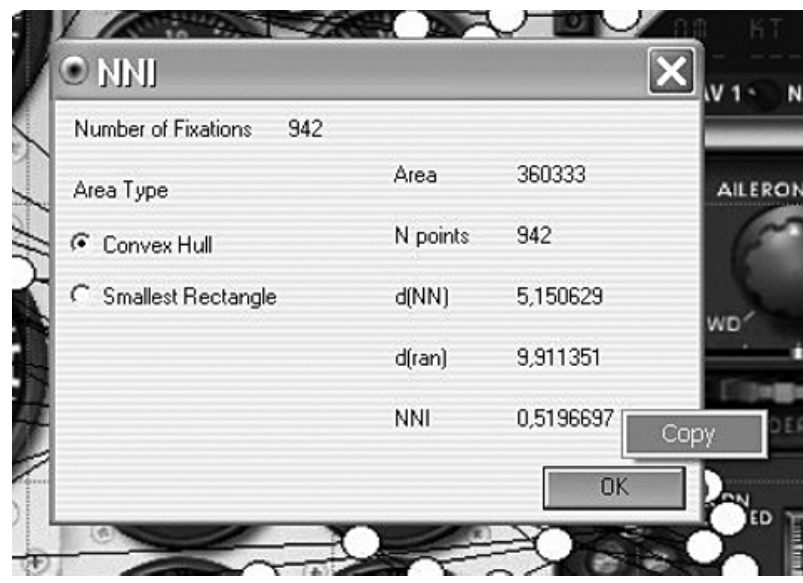

Figure 4. Example of the nearest neighbor index (NNI) computation results on one area of interest.

where $A$ is the study area and $B$ is the length of the perimeter of the study area.

In order to investigate the visual scanning behavior via the $R$ analysis, the choice to use the $\mathrm{CH}$ rather than the SR (or vice versa) could be based on methodological assumptions of the researchers only.

The release of ASTEF that is described in this article also implements the $R$ time series analysis. This function is available from the "Analyze" menu. This type of analysis, as briefly discussed in the Introduction section, could be very useful for obtaining continuous information about individuals' ocular behavior. More specifically, the "time series" function asks the user to insert the interval (in milliseconds) that represents the epoch within which $R$ is calculated.

The time series output provides an ASCII tab-separated data file that includes the following information:

A

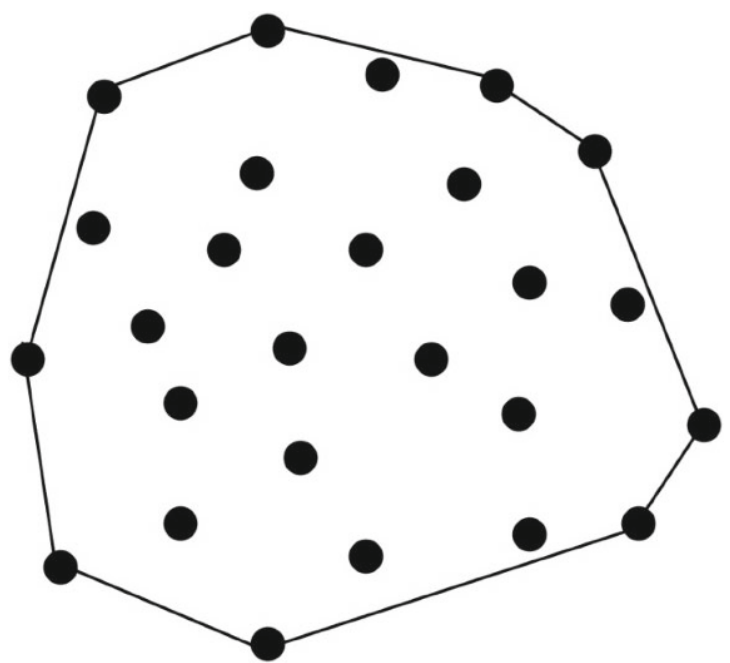

1. number of observation: number of the epoch within the whole recording time;

2. number of fixations within the matching epoch;

3. $R$ value within the matching epoch, computed using the $\mathrm{CH}$ area;

4. $R$ value within the matching epoch, computed using the SR area;

5. mean fixation duration within the matching epoch; and

6. median fixation duration within the matching epoch.

\section{Fixations Identifier Tool}

The analysis of fixations requires, as a first step, manipulation of the raw gaze data for identifying location and duration of each fixation point. This reduction allows minimizing the complexity of raw eye-movement data by collapsing gaze points sets in one or more fixation points.

It is a generally accepted fact that visual/cognitive processes occur during fixation (see, e.g., Just \& Carpenter, 1984). Indeed, no visual processing can be obtained during a saccade (Goldberg \& Schryver, 1995), and small eye movements (e.g., tremors, drifts, and flicks) are unimportant for higher level cognitive analyses (Alpern, 1962). Nevertheless, identifying fixation locations from raw gaze data still appears to be a subjective process, and poor definition of fixation identification algorithms might end in too many or too few fixations. An algorithm might also be too sensitive to the presence of outlier gaze points that could bias the interpretation of visual search paths (see also Salvucci \& Goldberg, 2000).

This section describes the tool for identifying fixations that is implemented in ASTEF. Its identification process is a space/time-based method that separates fixation and

Figure 5. (A) Convex hull and (B) smallest rectangle are two types of envelopes for point patterns. The figure shows the two types over the same point pattern.

B

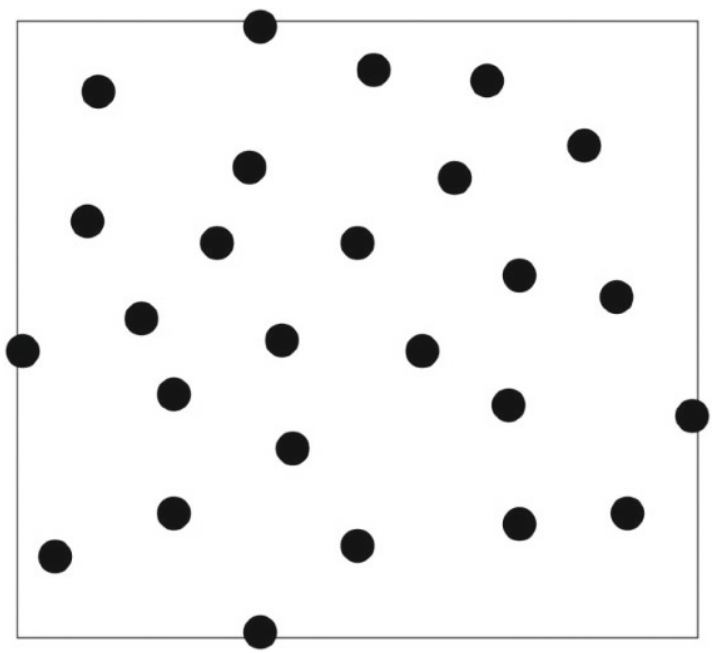


While there are still points

Initialize circumference over first point to cover the MinFixDuration

If distance of points from circumference center <- Radius Blse

Add additional points to circumference until duration $=>$ MinFixDuration

Do not remove points

until duration $\Rightarrow>$ MinPixDuration

//Noise Filter checks

Note a fixation at the centroid of the circumference points

Remove circumference points from points

Remove first point from points

Return fixations

Figure 6. Pseudocode for the fixation identifier algorithm, adapted from Salvucci and Goldberg (2000, p. 74).

saccade points on their point-to-point location and permanence inside that location.

The "Fixation Identifier" tool is available from the "Tools" menu. Figure 6 illustrates (in pseudocode form) the identification algorithm process.

In order to obtain fixations, the user needs to set two parameters: "Min Fixation Duration" (in milliseconds), which is the minimum duration of the fixation, and "Radius" (in pixels), which is the minimum fixation radius. The latter is nothing more than the projection on the screen of the threshold visual angle. Default values are those frequently reported in the literature (Hornof \& Halverson, 2002; Jacob \& Karn, 2003; Jainta \& Jaschinski, 2002; Kramer \& McCarley, 2003; Salvucci \& Goldberg, 2000): $0.5^{\circ}$ to $1^{\circ}$ of visual angle and $100-200$ msec of duration.

A noise filter is also implemented, because sporadic points falling outside the fixations may be found during the identification process (Alpern, 1962; Ditchburn, 1980; Hornof \& Halverson, 2002). Sometimes, after a first outlier, several other points may fall into the identified fixation. Ignoring those points may cause a biased estimate; for this reason, a noise filter has been implemented in order to check for the timing of those points occurring after the outlier point.

Given that the quality of a recorded gaze is affected by many factors (e.g., head movements, glasses, and contact lenses), ASTEF also implements two columns in the gaze data files that refer to the sampling quality (one for each eye). A 0 to 4 validity range is used, where 0 represents the best tracking quality. However, this information is not fully processed in the present release of the software (as of this article's publication), and only those gazes having the maximum tracking validity ( 0 coded) are taken into consideration. Such strictness is necessary due to the fact that lower validity reflects a lack of information about some features of the gaze (e.g., either the eye coordinates are missing or they are poorly precise), and it is our opinion that it is much more appropriate to exclude those samples. Nevertheless, future versions will implement a userdefined rejection threshold.

The last column of each ASTEF gaze data file is named "Flag." This could contain additional information (e.g., number of the participant, condition, and trial). This column is processed by the fixation identifier algorithm and by reducing a gaze data file if any information is lost. In brief, the algorithm signs a noted fixation with the description of its first gaze (i.e., matching with the fixation start time).

In order to identify a list of fixations, a gaze data file properly formatted (i.e., ASCII tab-separated) should be opened.

Fixation identifier input files include the following information:

1. timestamp - timing information about each recorded gaze;

2. pupil-pupil diameter information for each eye $(s x, d x)$

3. validity codes - gaze recording quality for each eye $(s x, d x)$;

4. gaze point - pixel coordinates $(x, y)$ of the gaze location in the area (origin is located at the upper left-hand corner); and

5. flag - optional information that can be added by the experimenter for defining the experimental condition to which the gaze belongs.

\section{ASTEF VALIDATION}

In order to validate the ASTEF, its results have been compared with the results obtained through the use of other software. The ASTEF identification process for detecting fixation data from a raw gaze data file has been compared with that of ClearView (Tobii Technology, Sweden) and of iComponent (Špakov, 2006a). The first is a commercial software suite, and the second is open-source software.

The ASTEF NNI $(R)$ function has been compared with paleontological statistics (PAST; Hammer, Harper, \& Ryan, 2001), a free data analysis package used in paleontology that includes a module for computing the $R$ statistics.

\section{Fixations}

The fixation data obtained by the fixation identifier tool in ASTEF have been compared with the outputs of ClearView and of the fixations detector tool (Špakov, 2006 b) implemented in iComponent. Sample gaze raw data from a task in which individuals freely explored a picture were used. An eyetracker device at $33 \mathrm{~Hz}$ was used for collecting eye-movement data on a 4:3, 17-in. display having a $1,024 \times 768$ pixel resolution. The identification algorithms of the three different packages were all based on spatial-temporal threshold parameters. In all three identifications, the algorithms' values were the projection of $1^{\circ}$ visual angle - which, at an approximate distance of $50 \mathrm{~cm}$, was equivalent to about a 25-pixel radius - and the minimal fixation duration of $100 \mathrm{msec}$. The three scanpaths (Figure 7) generated by the three different fixation identifications showed two minimal differences between them: (1) ClearView and iComponent detected one fixation more than did ASTEF, and (2) ASTEF and iComponent detected one fixation less than did ClearView. 


\section{A}

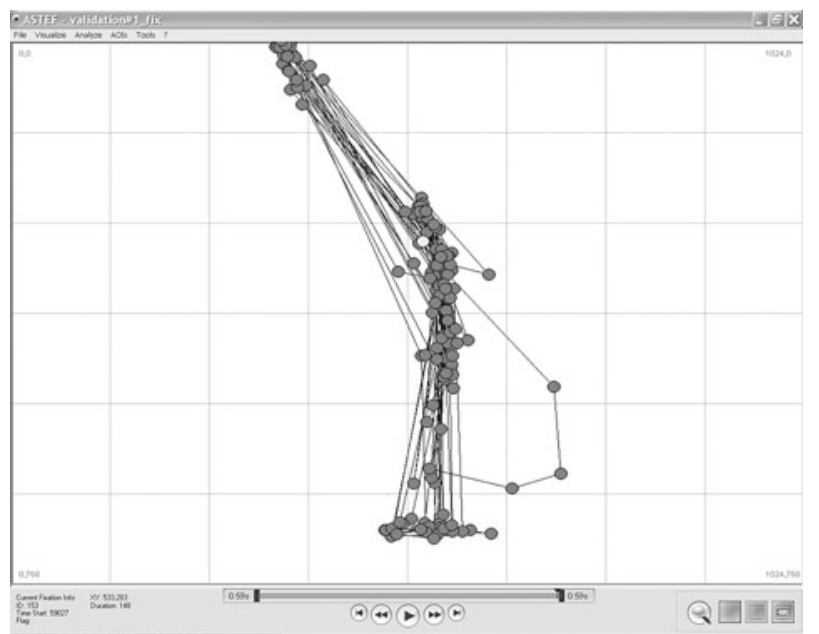

B

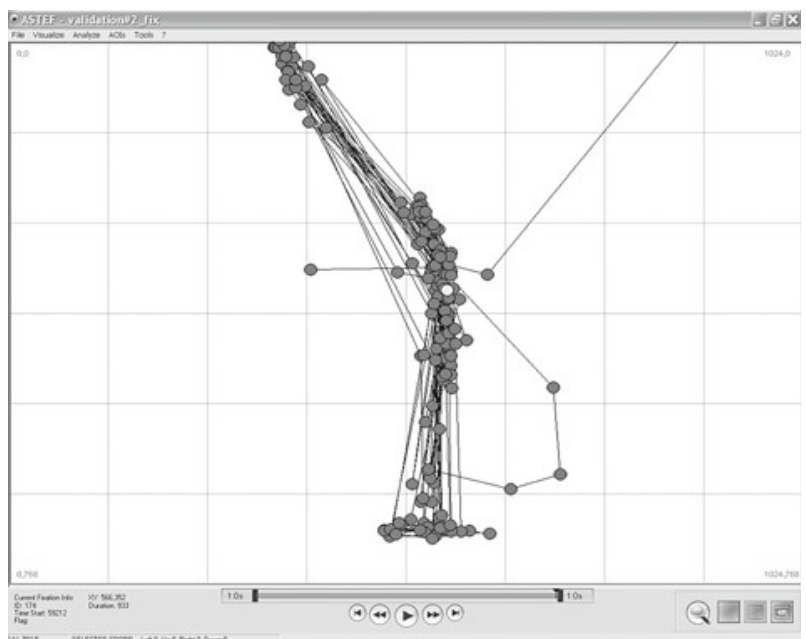

\section{C}

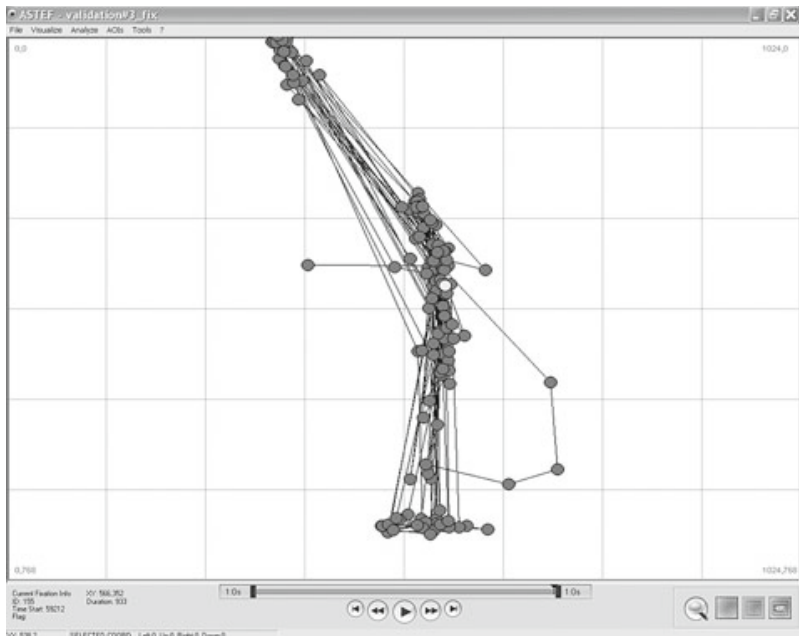

Figure 7. Scanpaths obtained by (A) ASTEF, (B) ClearView, and (C) iComponent.

These small discrepancies were probably due to negligible differences in the approximation of the fixation centroid - namely, in the calculation of the distance between each gaze and the center of the current fixation.

Moreover, while ASTEF and iComponent implement very similar noise filters, ClearView does not implement any filter in detecting fixations. This aspect is clearly observable from the comparison of the three different sets of fixation data. In fact, the inspection of the ClearView fixation output showed a higher number of fixations (174) and smaller durations (mean $=271 \mathrm{msec}$ ) than did ASTEF and iComponent outputs: 153 and 155 fixations, and 296and 299-msec mean durations, respectively.

Overall, the comparison shows the validity of the output obtained with the fixation identifier tool implemented in ASTEF.

\section{NNI}

As reported above, the spatial statistics function of ASTEF has been validated by comparison with PAST. PAST is a data analysis package that includes several functions that are commonly used in paleontology and ecology; it does not, therefore, provide any specific function for scanpath analysis. The same fixation data used for validating the fixation identification tool were used in this validation. Comparisons were made using both the $\mathrm{CH}$ and the SR algorithms. Donnelly's edge correction was implemented by default.

The results (Table 1 ) showed that the $R$ values were very similar. Particularly, $R$ values were exactly the same when using the SR area, and they were only slightly different when using the $\mathrm{CH}$ area. The negligible differences in the $\mathrm{CH}$ option were probably due to differences in approximation. 
Table 1

Comparison Between A Simple Tool for Examining Fixations (ASTEF) and Paleontological Statistics (PAST) in Computing the Nearest Neighbor Index From One Set of Fixation Data Using Both Convex Hull Area and Smallest Rectangle Area

\begin{tabular}{ccc}
\hline & \multicolumn{2}{c}{$\begin{array}{c}\text { Computation } \\
\text { Software Package }\end{array}$} \\
\cline { 2 - 3 } Type of Area & ASTEF & PAST \\
\hline Convex hull & .59060 & .57852 \\
Smallest rectangle & .40447 & .40447 \\
\hline
\end{tabular}

Validity of the nearest neighbor analysis output was confirmed, showing ASTEF to be a good candidate for the analysis of the spatial distribution of fixations (using the $R$ algorithm). Indeed, ASTEF provides clear and straightforward access to the data. For ease of use, all of the analysis functions can be accessed by right-clicking on the screen selection, as well as from the "Analyze" menu. Results appearing in the dialog box can be copied (by right-clicking on them) and then pasted into documents or spreadsheets.

\section{CONCLUSIONS}

ASTEF works with fixation data recorded by any eyetracking system, provided those data are properly formatted (i.e., as an ASCII tab-separated file). Although ASTEF can import previously identified fixations and their locations, it can also import raw ocular data and locate the fixations using user-defined parameters. The tool for identifying fixations makes this software a valuable resource for the researcher.

In developing ASTEF, our first aim was to provide companion software for encouraging the use of novel methods for the analysis of fixations in the HF/E domain, but we also wanted to provide a fast and usable piece of software. The graphical user interface has been designed to make available to users a complete visualization of the scanpath and direct access to the analysis functions. ASTEF also allows researchers to directly manipulate the visualized scanpath. This functionality is considered to be one of the most important features in information visualization systems, and the program offers the opportunity to manipulate points (i.e., color and size), define AOIs, and obtain information with mouse clicks. Its main advantage is that it allows easy loading, managing, saving, and exporting of scanpaths. A small set of analysis functions was also implemented, and it is very easy to gather information about the number of fixations in an AOI, as well as their mean duration (two variables often used by researchers).

The NNI calculation for assessing the type of distribution (i.e., distribution of fixations) was also implemented and validated. The method has been supported by recent reports (Camilli et al., 2007; Di Nocera et al., 2006; Di Nocera et al., 2007) indicating a strict relationship between the spatial distribution of fixations and the mental workload experienced by the participants. The variations of the index in time can also be easily assessed, because the time series analysis has been also implemented. The latter function was added during the writing of the present article, and future plans have been made to add a complete set of techniques suited for fixation spatial distribution analysis in the HF/E domain.

\section{AUTHOR NOTE}

This work was supported by the European Office of Aerospace Research and Development, United States Air Force Office of Scientific Research, Grant 053021. The authors thank Oleg Špakov at the University of Tampere, Finland, for his help with the fixation identification algorithm. Correspondence concerning this article should be addressed to F. Di Nocera, Cognitive Ergonomics Laboratory, Department of Psychology, University of Rome "La Sapienza," Via dei Marsi 78-00185, Rome, Italy (e-mail: dinocera@uniroma1.it).

\section{REFERENCES}

AlPern, M. (1962). Type of movement. In H. Davson (Ed.), The eye: Vol. 3. Muscular mechanisms (pp. 63-151). New York: Academic Press.

Bellenkes, A. H., Wickens, C. D., \& Kramer, A. F. (1997). Visual scanning and pilot expertise: The role of attentional flexibility and mental model development. Aviation, Space, \& Environmental Medicine, 68, 569-579.

BUNECKE, J. L. (1987). Quantifying some information processing requirements of the pilot's instrument crosscheck. Proceedings of the Human Factors Society Annual Meeting, 31, 1301-1305.

Camilli, M., Terenzi, M., \& Di Nocera, F. (2007). Concurrent validity of an ocular measure of mental workload. In D. de Waard, G. R. J. Hockey, P. Nickel, \& K. A. Brookhuis (Eds.), Human factors issues in complex system performance (pp. 117-129). Maastricht: Shaker.

Clark, P. J., \& Evans, F. C. (1954). Distance to nearest neighbor as a measure of spatial relationships in populations. Ecology, 35, 445-453.

Delaunay, B. (1934). Sur la sphère vide [On the empty area]. Izvestia Akademii Nauk SSSR, Otdelenie Matematicheskikh i Estestvennykh Nauk, 7, 793-800.

Diez, M., Boenm-Davis, D. A., Holt, R. W., Pinney, M. E., HansBERGER, J. T., \& SCHOPPEK, W. (2001). Tracking pilot interactions with flight management systems through eye movements. Proceedings of the 11th International Symposium on Aviation Psychology (pp. 1-6). Columbus: Ohio State University.

Di Nocera, F., \& Bolia, R. S. (2007). PERT networks as a method for analyzing the visual scanning strategies of aircraft pilots. Proceedings of the 14th International Symposium on Aviation Psychology (pp. 165-169). Dayton, OH: Wright State University.

Di Nocera, F., Camilli, M., \& Terenzi, M. (2007). A random glance at the flight deck: Pilots' scanning strategies and the real-time assessment of mental workload. Journal of Cognitive Engineering \& Decision Making, 1, 271-285.

Di Nocera, F., Terenzi, M., \& Camilli, M. (2006). Another look at scanpath: Distance to nearest neighbour as a measure of mental workload. In D. de Waard, K. A. Brookhuis, \& A. Toffetti (Eds.), Developments in human factors in transportation, design, and evaluation (pp. 295-303). Maastricht: Shaker.

DitchiuRn, R. W. (1980). The function of small saccades. Vision Research, 20, 271-272.

DonNelLy, K. P. (1978). Simulations to determine the variance and edge effect of total nearest-neighbor distance. In I. Hodder (Ed.), Simulation studies in archaelogy (pp. 91-95). Cambridge: Cambridge University Press.

Ellis, S. R., \& Smith, J. D. (1985). Patterns of statistical dependency in visual scanning. In R. Groner, G. W. McConkie, \& C. Menz (Eds.), Eye movements and human information processing (pp. 221-238). Amsterdam: Elsevier.

Ephrath, A. R., Tole, J. R., Stephens, A. T., \& Young, L. R. (1980). Instrument scan-Is it an indicator of the pilot's workload? Proceedings of the Human Factors Society Annual Meeting, 24, 257-258.

GoldberG, J. H., \& Schryver, J. C. (1995). Eye-gaze-contingent control of the computer interface: Methodology and example for zoom detection. Behavior Research Methods, Instruments, \& Computers, 27, 338-350. 
Hammer, Ø., Harper, D. A. T., \& Ryan, P. D. (2001). PAST: Paleontological statistics software package for education and data analysis. Palaeontologia Electronica, 4. Retrieved April 5, 2006, from palaeo-electronica.org/2001_1/past/main.htm; available from folk .uio.no/ohammer/past/.

Harris, R. L., Glover, B. L., \& Spady, A. A. (1986). Analytic techniques of pilot scanning behavior and their application (NASA Tech. Rep. No. 2525). Hampton, VA: Langley Research Center.

Hilburn, B., Jorna, P. G., Byrne, E. A., \& Parasuraman, R. (1997) The effect of adaptive air traffic control (ATC) decision aiding on controller mental workload. In M. Mouloua \& J. M. Koonce (Eds.), Human-automation interaction: Research and practice (pp. 84-91). Mahwah, NJ: Erlbaum.

Hornof, A. J., \& Halverson, T. (2002). Cleaning up systematic error in eye-tracking data by using required fixation locations. Behavior Research Methods, Instruments, \& Computers, 34, 592-604.

JACOB, R. J. K., \& KARN, K. S. (2003). Eye tracking in human-computer interaction and usability research: Ready to deliver the promises (Section Commentary). In J. Hyönä, R. Radach, \& H. Deubel (Eds.), The mind's eye: Cognitive and applied aspects of eye movement research (pp. 573-605). Amsterdam: Elsevier.

JAINTA, S., \& JASCHINSKI, W. (2002). Fixation disparity: Binocular vergence accuracy for a visual display at different positions relative to the eyes. Human Factors, 44, 443-450.

Just, M. A., \& CARPEnTER, P. A. (1984). Using eye fixations to study reading comprehension. In D. E. Kieras \& M. A. Just (Eds.), New methods in reading comprehension research (pp. 151-182). Hillsdale, NJ: Erlbaum.

Kasarskis, P., Stehwien, J., Hickox, J., Aretz, A., \& Wickens, C. (2001). Comparison of expert and novice scan behaviors during VFR flight. In Proceedings of the 11th International Symposium on Aviation Psychology. Retrieved October 4, 2006, from www.humanfactors .uiuc.edu/Reports\&PapersPDFs/isap01/proced01.pdf.

Kramer, A. F., \& MCCARley, J. S. (2003). Oculomotor behaviour as a reflection of attention and memory processes: Neural mechanisms and applications to human factors. Theoretical Issues in Ergonomics Science, 4, 21-55.

KruizingA, A., Mulder, B., \& DE WAARd, D. (2006). Eye scan patterns in a simulated ambulance dispatcher's task. In D. de Waard, K. A. Brookhuis, \& A. Toffetti (Eds.), Developments in Human Factors in
Transportation, Design, and Evaluation (pp. 305-317). Maastricht: Shaker.

McCarley, J. S., \& Kramer, A. F. (2007). Eye movements as a window on perception and cognition. In R. Parasuraman \& M. Rizzo (Eds.), Neuroergonomics: The brain at work (pp. 95-112). New York: Oxford University Press.

Miller, J. M. (1973). Visual behavior changes of student pilots flying instrument approaches. Proceedings of the Human Factors Society Annual Meeting, 17, 208-214.

Noton, D., \& StARK, L. (1971). Scanpaths in saccadic eye movements while viewing and recognizing patterns. Vision Research, 11, 929-942.

Ponsoda, V., ScotT, D., \& Findlay, J. M. (1995). A probability vector and transition matrix analysis of eye movements during visual search. Acta Psychologica, 88, 167-185.

Recarte, M. A., \& Nunes, L. M. (2000). Effects of verbal and spatialimagery tasks on eye fixations while driving. Journal of Experimental Psychology: Applied, 6, 31-43.

Salvucci, D. D., \& GoldberG, J. H. (2000). Identifying fixations and saccades in eye-tracking protocols. Proceedings of the Eye Tracking Research and Applications Symposium (pp. 71-78). New York: ACM Press.

ŠPakov, O. (2006a). iComponent v. 3.28. Retrieved October 24, 2006 , from www.cs.uta.fi/ oleg/icomp.html.

Špakov, O. (2006b). Fixation Detector v. 1.20. Retrieved November 11, 2006, from www.cs.uta.fi/ oleg/fixdet.html.

STARK, L. W., \& ElLIS, S. R. (1981). Scanpaths revisited: Cognitive models direct active looking. In D. F. Fisher, R. A. Monty, \& J. W. Senders (Eds.), Eye movements: Cognition and visual perception (pp. 193-226). Hillsdale, NJ: Erlbaum.

Tole, J. R., Stephens, A. T., Vivaudou, M., Ephrath, A. R., \& Young, L. R. (1983). Visual scanning behavior and pilot workload (NASA Contractor Rep. No. 3717). Hampton, VA: Langley Research Center.

VAn Orden, K. F., Limbert, W., MaKeig, S., \& Jung, T.-P. (2001). Eye activity correlates of workload during a visuospatial memory task. Human Factors, 43, 111-121.

(Manuscript received September 29, 2006; revision accepted for publication August 3, 2007.)

\section{Notice}

The articles from the 2007 meeting of the Society for Computers in Psychology (SCiP) will be published in the August 2008 issue of Behavior Research Methods. 